\title{
EDITORIAL
}

\section{Epithelial-to-mesenchymal transition and cancer stem cells: emerging targets for novel cancer therapy}

Cancer Gene Therapy (2014) 21, 179-180; doi:10.1038/cgt.2014.17

Most cancer patients die from metastatic disease and recurrences following conventional cancer therapies, including chemotherapy and radiotherapy. Cancer stem cells (CSCs), which have the capacity for extensive proliferation, self-renewal, multi-lineage differentiation and high tumorigenic potential, have been found in most types of solid and hematopoietic tumors. CSCs show a high propensity for invasiveness and metastasis, and are found to be resistant to conventional cancer therapies. Emerging evidence suggests that there is a strong relationship between CSCs and epithelial-to-mesenchymal transition (EMT) in most carcinomas. In this issue, Findlay et al. ${ }^{1}$ present a review that highlights the recent evidence linking EMT with aggressive tumor biology as well as with the CSCs across multiple organ systems focusing on colon cancer. As we continue to lack novel strategies overcoming metastatic disease and therapeutic resistance, EMT and CSC molecular pathways may reveal and highlight novel therapeutic targets.

EMT is a set of biochemical changes that result in epithelial cells acquiring a pluripotent mesenchymal phenotype, including enhanced migratory capacity, invasiveness, elevated resistance to apoptosis and increased production of extracellular matrix components. $^{2}$ EMT is characterized by loss of cell adhesion (typically represented by downregulation of E-cadherin), acquisition of mesenchymal markers (including $\mathrm{N}$-cadherin, Vimentin, Fibronectin and transcription factors) and increased cell motility. ${ }^{2}$ EMT in cancer is believed to enhance metastasis because of the increased migratory and invasive capacity of mesenchymal cells. ${ }^{3,4}$ The zinc-finger transcription factors SNAIL, SLUG, ZEB1 and TWIST, repress genes responsible for the epithelial phenotype and induce genes responsible for the mesenchymal phenotype, thereby representing important regulators of EMT. ${ }^{2}$

Both EMT and CSCs play critical roles in cancer metastasis and therapeutic resistance, and they share molecular characteristics. ${ }^{3} \mathrm{~A}$ strong association between EMT-associated gene expression and
CSCs has been described by Findlay et al. For example, both transforming growth factor- $\beta$ treatment and expression of EMT transcription mediators such as SNAIL in mammary epithelial cells or colon cancer cells demonstrated CSC phenotype. CD44 ${ }^{+}$/ $\mathrm{CD}_{24}^{-}$breast cancer cells consistently had higher EMT gene expression patterns than more differentiated cancer cells. Negative feedback loops exist between the microRNA 200 (miR200) family, and the EMT transcription factors as well as CSC phenotype. The miR-200 family repressed EMT transcription factors such as ZEB1, and ZEB1 repressed miR-200 family members. The miR-200 family inhibited the growth and phenotype of CSCs and was down-regulated in CSCs. Currently, agents targeting CSC unique mediators have demonstrated preclinical success and have already been used in clinical trials. ${ }^{5}$ As EMT promotes CSC formation, targeting EMT in broad cancer histologies may be a promising therapeutic strategy along with CSC-targeting agents.

Various signaling pathways can induce EMT. Alterations in genes associated with developmental pathways such as WNT, Hedgehog and NOTCH are common in various cancers and have been shown to facilitate EMT. ${ }^{2}$ WNT signaling comprises both canonical and non-canonical pathways. ${ }^{6}$ The non-canonical pathway is $\beta$-cateninindependent, whereas the canonical pathway is regulated by $\beta$-catenin. In the absence of WNT ligands, $\beta$-catenin levels are regulated by a multiprotein complex encompassing kinases (glycogen synthase kinase-3 $\beta$ (GSK3 $\beta$ ) and casein kinase 1 (CK1)) and scaffolding proteins (adenomatous polyposis coli, Axin1 and Axin2). This 'destruction complex' binds and phosphorylates $\beta$-catenin at $\mathrm{N}$-terminal Ser and Thr residues, thus targeting it for ubiquitination and proteolytic degradation. In the presence of WNT ligands, co-activation of the Frizzled and low-density lipoprotein receptor-related protein (LRP) receptors inhibits the destruction complex and stabilizes $\beta$-catenin. Cytoplasmic $\beta$-catenin accumulation results in its nuclear translocation. In the nucleus, $\beta$ catenin activates transcription of WNT/ $\beta$-catenin target genes. Aberrations in the WNT pathway leading to elevated $\beta$-catenin as well as mutations in the genes involved in the WNT pathway are quite common in various cancer histologies. ${ }^{7}$ Cancer cell invasion
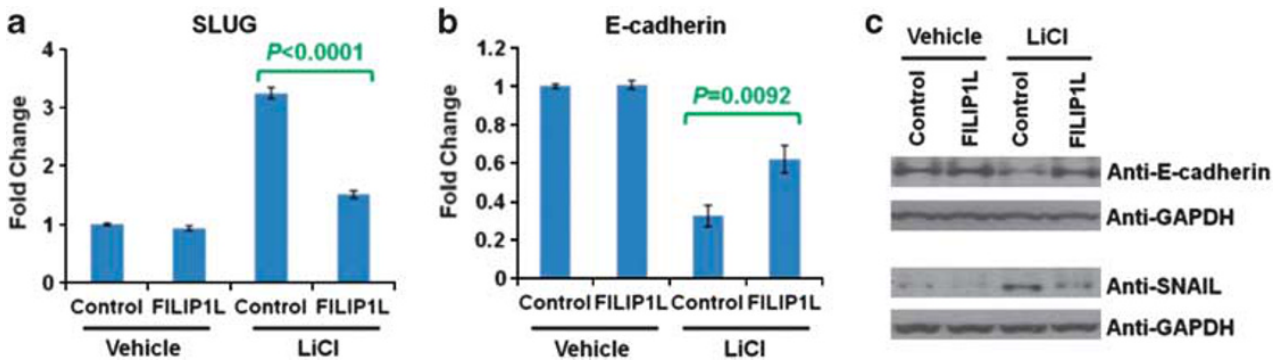

Figure 1. FILIP1L inhibited repression of epithelial markers and induction of mesenchymal markers following activation of WNT signaling in pancreatic cancer cells. Quantitative reverse transcription-PCR analysis for SLUG (a) and E-cadherin (b) conducted on cDNA from MIA PaCa-2 (a) or PANC-1 (b) cells. Cells were transfected with either control or FILIP1LAC103 cDNA followed by either vehicle or $40 \mathrm{~mm}$ LiCl treatment. The $y$ axis represents fold change over vehicle-treated, uninduced cells (Vehicle-CTL), where each value was standardized with the housekeeping gene GAPDH. Error bars indicate s.e.m. $(n=3)$. (c) Immunoblot analysis for E-cadherin (from PANC-1 cell lysates) and SNAIL (from MIA PaCa-2 cell lysates) from the same cells utilized in panels $\mathbf{a}$ and $\mathbf{b}$. GAPDH blot is shown as the loading control. 
and metastasis are shown to be mediated by an activated WNT/ $\beta$ catenin signaling pathway. ${ }^{7}$

An activated canonical WNT pathway facilitates EMT in a number of ways: $^{8}$ WNT/ $\beta$-catenin upregulates mesenchymal markers, including SLUG, ZEB1 and TWIST, which in turn repress $E$-cadherin. There is also an increase in matrix metalloproteinases (MMPs), such as MMP3, -7 and -9 , which modulate the extracellular matrix, enhance invasion and inactivate E-cadherin by cleaving its ectodomain. Finally, WNT signaling leads to increased levels of SNAIL by inhibiting its phosphorylation by GSK3 $\beta$ that destines it for degradation. A plethora of evidence therefore supports a cascade of events, wherein WNT signaling activates EMT and promotes cancer metastasis.

Therefore, finding a new target for WNT signaling can contribute to the EMT-targeted therapeutic strategy. Our laboratory has identified a novel tumor suppressor-like protein, Filamin A interacting protein 1-like (FILIP1L), as an important inhibitor of cell migration and invasion. 9,10 We showed that FILIP1L, like many other tumor suppressors, is downregulated by promoter hypermethylation in various cancers such as ovarian, pancreatic, breast, colon and lung. ${ }^{9,10}$ We also showed that FILIP1L inhibits ovarian cancer metastasis by inhibiting WNT signaling. ${ }^{11}$ FILIP1L also inhibited EMT following WNT pathway activation in pancreatic cancer cells. Upon WNT pathway activation, FILIP1L significantly inhibited repression of E-cadherin and induction of SNAIL and SLUG in these pancreatic cancer cells (Figure 1). We also observed a similar phenomenon in ovarian cancer cells (data not shown). Currently, we are actively investigating whether EMT inhibition by FILIP1L contributes to its inhibition of metastases in these cancer types.

FILIP1L may represent a new target for the EMT pathway. Finding new molecules involved in EMT and CSC pathways as well as exploring specific drugs targeting these molecules will serve novel therapeutic strategies for improving the response to conventional anti-neoplastic agents.

\section{CONFLICT OF INTEREST}

The authors declare no conflict of interest.

M Kwon

Albert Einstein College of Medicine, Bronx, NY, USA

E-mail: mkwon@einstein.yu.edu

\section{REFERENCES}

1 Findaly VJ, Wang C, Watson DK, Camp ER. Epithelial to mesenchymal transition and cancer stem cell phenotype: insights from cancer biology with therapeutic implications for colorectal cancer. Cancer Gene Ther 2014; 21: 181-187.

2 Kalluri R, Weinberg RA. The basics of epithelial-mesenchymal transition. J Clin Invest 2009; 119: 1420-1428.

3 Polyak K, Weinberg RA. Transitions between epithelial and mesenchymal states: acquisition of malignant and stem cell traits. Nat Rev Cancer 2009; 9: 265-273.

4 Tsai JH, Yang J. Epithelial-mesenchymal plasticity in carcinoma metastasis. Genes Dev 2013; 27: 2192-2206.

5 Saeki T, Nomizu T, Toi M, Ito Y, Noguchi S, Kobayashi T et al. Dofequidar fumarate (MS-209) in combination with cyclophosphamide, doxorubicin, and fluorouracil for patients with advanced or recurrent breast cancer. J Clin Oncol 2007; 25: 411-417.

6 Clevers H, Nusse R. Wnt/beta-catenin signaling and disease. Cell 2012; 149 1192-1205.

7 Polakis P. Drugging Wnt signalling in cancer. EMBO J 2012; 31: 2737-2746.

8 Heuberger J, Birchmeier W. Interplay of cadherin-mediated cell adhesion and canonical Wnt signaling. Cold Spring Harb Perspect Biol 2010; 2: a002915.

9 Burton ER, Gaffar A, Lee SJ, Adeshuko F, Whitney KD, Chung JY et al. Downregulation of Filamin A interacting protein 1-like is associated with promoter methylation and induces an invasive phenotype in ovarian cancer. $\mathrm{Mol}$ Cancer Res 2011; 9: 1126-1138.

10 Kwon M, Lee SJ, Reddy S, Rybak Y, Adem A, Libutti SK. Down-regulation of Filamin A interacting protein 1-like is associated with promoter methylation and an invasive phenotype in breast, colon, lung and pancreatic cancers. PLOS ONE 2013; 8: e82620.

11 Kwon M, Lee SJ, Wang Y, Rybak Y, Luna A, Reddy S et al. Filamin A interacting protein 1-like inhibits WNT signaling and MMP expression to suppress cancer cell invasion and metastasis. Int J Cancer 2014; 135: 48-60. 